\title{
Influence of Road Geometry on Driver's Gaze Behavior on Motorways
}

\author{
Borislav Hristov ${ }^{*}$ \\ University of Applied Sciences Berlin, Germany
}

\begin{abstract}
Driver's gaze is a highly selective dynamic process, which ensures a precise perception of moving objects, speeds and spatial expansions in road traffic. Thus, the gaze behavior of drivers determines largely their driving behavior, and is crucial for traffic safety. With the aid of modern technology, the gaze behavior can be precisely recorded during driving. This paper describes part of the research, which has been undertaken in order to investigate the relationship between driver's gaze behavior and horizontal alignment geometry. It is known, that driver's stress state and gaze concentration are not the same when steering a vehicle along straight stretches and within curves. The stress is higher in curves and this leads to an increased attention. The gaze behavior in left and right horizontal curves is characterized by certain gaze patterns, which clearly differ from each other. In this respect, appropriate investigations and analyzes have been also carried out. Different parameters have been calculated in order to assess and quantify the gaze behavior. By evaluating these parameters, the spatial and temporal concentration of the driver's gaze as well as the intensity of information acquisition and processing have been analyzed along straight stretches, left and right horizontal curves.
\end{abstract}

\section{Introduction}

Over $90 \%$ of the information by driving is acquired through the visual sense channel. The eye is the only sense organ, which can be aligned selectively through the eye movements and allows the detection and localization of movable and immovable objects. Thus, the gaze behavior of drivers plays a central role in the safe steering of vehicles. Since information reception and processing are the basis for action decisions, their realization and control in road traffic, the driver's gaze behavior determines largely his driving behavior, and is thus crucial for traffic safety. Current modern measurement and computing technology allows a highly accurate capture of the driver's gaze movements by determining the exact gaze direction, coordinates of each focal point, the beginning and the end of each fixation and the calculation of many other gaze behavior parameters. Thereby, the analysis of the gaze behavior is a method, by which the perceptual psychological factor in the interaction between driver, vehicle and road can be considered. Accordingly, it is possible to investigate how the driver's gaze behavior is influenced by the horizontal alignment geometry. The influence of the road characteristics parameters on the driving behavior has

\footnotetext{
* Corresponding author: Borislav.Hristov@HTW-Berlin.de
} 
been a subject of numerous research work in the field of road design for a long time. On motorways, however, only a few such studies are available and there is still a lack of sufficient knowledge in this respect.

\section{Background}

Driver's gaze is a highly selective dynamic process which ensures a precise perception of moving objects, speeds and spatial expansions. The analysis of the visual behavior makes the investigation of the temporal as well as the spatial course of the detailed information flow possible.

The gaze behavior of motorists is determined to a considerable degree by the road geometry. It is known that driver's stress state and gaze concentration are not the same when steering a vehicle along straight sections and within horizontal curves. Driver's stress is higher in curves compared to straight sections and this leads to an increased attention. The gaze behavior in left and right horizontal curves is characterized by certain gaze patterns, which clearly differ from each other.

According to [1], the main fixation point for straight lines is about $0.7^{\circ}$ above and $1.6^{\circ}$ to the right of the vanishing point. The authors write that the fixation durations are longer in straight lines than in curves $(0.60 \mathrm{~s}$ vs. $0.41 \mathrm{~s})$ with a smaller gap between two consecutive fixations $\left(2.6^{\circ}\right.$ vs. $\left.3.1^{\circ}\right)$. They confirm the fact that on straight sections most of the views are aligned in the area of the vanishing point. This determines the direction and lateral position controlled by peripheral vision. These differences reflect from the more intense visual search activity within a curve compared to a straight line. On right curves the drivers are fixating $3.6^{\circ}$ to the right, on left curves, however, only $0.3^{\circ}$ to the left. It is noted that drivers fixate the road significantly longer on right curves $(55 \%)$ than in left curves $(38 \%)$. On right curves drivers look more to the right, whereas in left curves the gaze is orientated straight ahead and scatters more. While on right curves the road and the scenery are fixated on the left side for only $5 \%$ of the total time, the fixation time on the right side of the road in left turns is $24 \%$. Within the curves, the drivers fixate longer at the environment $(27 \%)$ than they do at the road (23\%).

In right curves, the right edge of the road is preferably fixed. In left curves, however, the fixations are distributed between the left lane edge and the center of the lane ([2], [3] and [4]). [5] concludes that the information is mainly obtained from the right side of the road. The fixation points are distributed over the entire width of the road at different distances in left curves. Current investigations by [6] prove that drivers fixate more than $2 / 3$ of the time at the vanishing point in straight sections. According to [7], the optical density between the tangent point (TP) and the vanishing point (VP) of the road plays a major role in the estimation of curvature and lane tracking. Accurate driving requires looking into the closeup and the far range of the road ([8]). The far range of the road is necessary for the preview of the curvature and the close-up range is important for the lane tracking. At low speeds, the close-up range of the road is sufficient for the correct driving style. Yorozu et al [9] investigate the gaze behavior in real driving and note that the fixations on straight sections are mainly in the far range of the road and less fixations land on the left and the right road edges with equal percentage. This distribution of fixations does not change significantly until $50 \mathrm{~m}$ before a curve. The number of fixations increases in an area of approx. $30 \mathrm{~m}$ before the curve and the distribution of fixations in the horizontal direction is concentrated on the respective inner side of the curve (left for left curves and right for right curves). [10] research the gaze behavior in a driving simulator on straight sections, left and right curves with radii of $2000 \mathrm{~m}$ and $5000 \mathrm{~m}$. The authors report that about $50 \%$ of the total fixation time is on the road. It is noted that in both straight lines and in curves, approximately $85 \%$ of all fixations lie in an angular range of $\pm 4^{\circ}$ in the horizontal and by $\pm 2^{\circ}$ in the vertical 
direction around the vanishing point (Figure 2.44). Therefore, the vanishing point is defined as an $8^{\circ}$ wide and $4^{\circ}$ high window.

\section{Method}

\subsection{Eye-tracking system}

The gaze behavior has been measured in real rides carried out by test persons using a modern high-tech measuring vehicle. To capture the gaze behavior, a vehicle was equipped with a highly accurate "Smart Eye Pro" system. This remote eye tracking system detects and records the eye movements according to the principle of corneal reflection. This reflection method (infrared oculography) is based on the registration of light reflexes on the corneal surface (cornea). The non-contact method for recording eye movements offers numerous advantages compared to the previously used head-based systems:

- The driver is not burdened by any weight or pressure.

- The driver's field of view is not restricted (large field of view).

- The head position is calculated by the system relative to the position of the vehicle on the road, thus eliminating the need for an additional complex calculation of the head position.

- The system works with glasses and sun glasses of non IR type.

- The system works in any lighting condition.

Two eye cameras with active infrared spots were attached to the windshield of the vehicle and detected the pupil movements (figure 1). The infrared spots of the two cameras were used to avoid disturbing effects from changing light conditions. An additional scene camera captured the driver's field of view from his perspective. The data from the eye movement measurement and the GPS data of the APPLANIX system for determining the position were processed in a central computer situated in the vehicle's trunk.

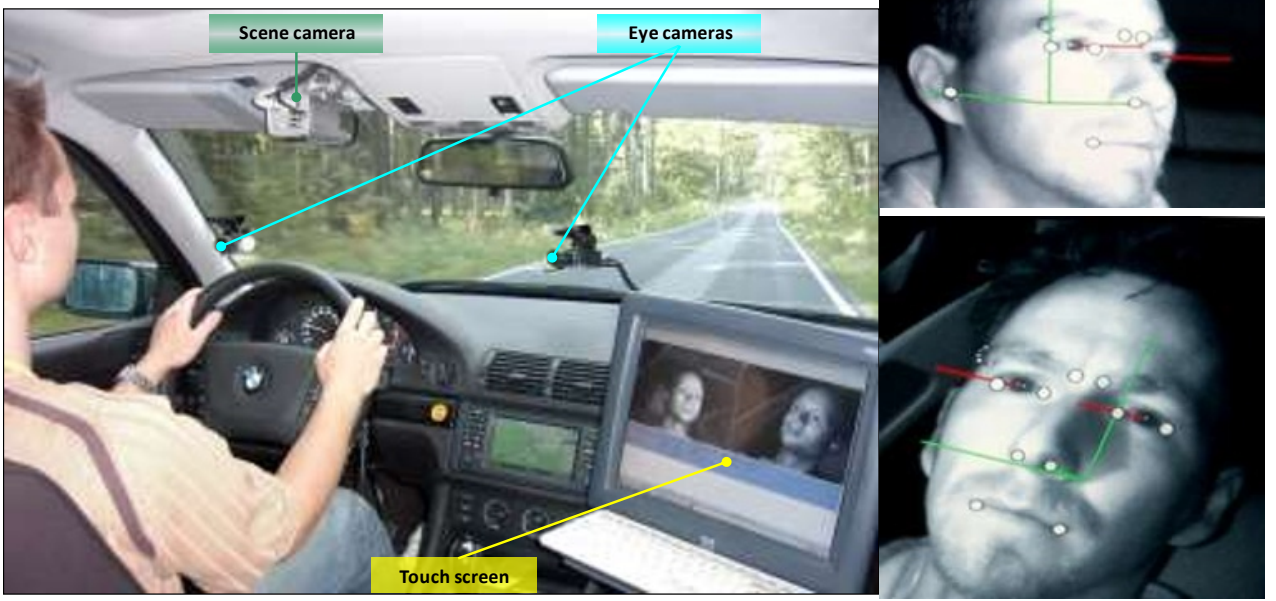

Fig. 1. Test vehicle with mounted measuring system for recording the eye movements of drivers. 


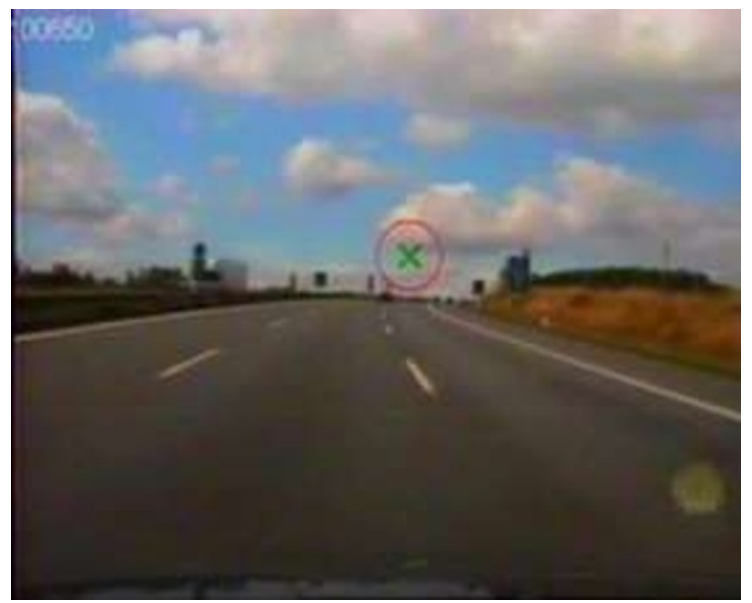

Fig. 2. Video image with recorded fixation point.

When calibrating the eye cameras, a head model was created for each subject. The head position was determined by 12 characteristic contrast points on the driver's head, which were marked on a touch screen next to the driver's seat (figure 1). These were the inner points of the eyebrows, the outer and inner points of the eyes, the nostrils, the left and right corners of the mouth, both ears and the centers of the pupils.

The accuracy of the model depends essentially on the exact positioning of the characteristic points. The program calculates the head model and the calculated points are then compared with the ones entered on the touch screen. Five shots of different head positions must be made for each driver in order to achieve a reference image of the head. On the touch screen in the car, the visual vectors emanating from the pupils and their movements are shown in real time by each of the two eye cameras (figure 1).

\subsection{Parameters of gaze behavior}

Simplified, the gaze behavior can be described as a chain-like continuing sequence of saccadic gaze movements and fixations. Through the change of saccades and fixations, the course of the information recording from the environment is reflected and the gaze behavior is defined. The saccadic gaze movements are very rapid, erratic rotational movements of the eyeball and they bring out blurred objects from the periphery for fixation on the fovea. The saccades reach a speed of up to $1000 \%$ s ([11] and [12]). Thus, the eye movements are the fastest movements that take place within the human body. Saccades are ballistic movements, this means as soon as a movement is triggered, course and destination cannot be corrected. The duration of a saccade lies in the range of 10 to $80 \mathrm{~ms}$, according to [12]. Each Saccade leads the eyes to a new fixation. The fixations and the gaze movements are the movements of the eyes that enable the information reception. During a fixation, the eyes are in relative standstill with respect to the viewed object. The fixation durations vary between 100 and $2000 \mathrm{~ms}$ and their concentration ranges between 200 and $600 \mathrm{~ms}$ ([13] and [14]). When analyzing fixation durations in road traffic, [15] states that fixations last between 300 and $400 \mathrm{~ms}$. The fixation periods are the shorter, the more "complex" the traffic situation is. Fixations on traffic signs take on average $500 \mathrm{~ms}$ and on the roadway $580 \mathrm{~ms}$.

\subsection{Time and spatial gaze behavior parameters}

The driver's gaze behavior can be analyzed using different gaze parameters, which indicate the spatial concentration of gaze behavior, the temporal behavior of visual attention 
distribution and the intensity of information reception (level of processing). On the one hand there are parameters of visual search, on the other - parameters of visual processing. In this research eight different parameters were used.

\section{Parameters of visual search}

- Scan path length $\left[^{\circ}\right]$ : spatial parameter that defines an optimal visual search based on reduction of saccade amplitudes. Scan path length is the sum of the distances between the scan viewpoints. It is an indicator for the efficiency of gaze behavior.

- Spatial density index per second [1/s]: Measure of the number of different places viewed (measure of gaze concentration).

- Saccades per second [1/s]: a large number of saccades in a scan path indicates an extensive visual search (measure of gaze concentration).

- Standard deviation of the gaze angle $\left[^{\circ}\right]$ : square root of the sum of the squared horizontal and vertical gaze angles (measure of gaze concentration).

\section{Parameters of visual processing}

- Number of fixations [-]: similar to the number of components the driver has to process, but not to the depth of processing.

- Average fixation duration [ms]: longer fixations indicate a longer interpretation time. Thus, the fixation duration is a measure of the depth of visual processing.

- Time share of fixations [-]: significantly higher with attentive than with inattentive gaze behavior. Therefore, it is interpreted as a measure of visual attention.

- Percent Road Centre (PRC) [-]: the percentage of all fixations directed to an area of $30^{\circ} \times 15^{\circ}$ around the road centre, also known as primary attention area (measure of visual orientation).

\subsection{Test track}

Test track was the A72 federal motorway between the cities Hof and Chemnitz, which is one of the oldest motorways in Germany and which is characterized by inhomogeneous route characteristics, was selected to investigate the visual behavior of drivers and accidents. The length was $56.0 \mathrm{~km}$ in each direction. The curves and straight lines were respectively $49.5 \%$ and $50.5 \%$ of the total length of the test section. Of these, $24.3 \%$ were left curves and $25.2 \%$ were right curves.

\subsection{Selection of the test person collective}

10 volunteers aged 26 to 39 of both sexes were selected. They were all required to have a driving experience of at least $60,000 \mathrm{~km}$. They should have held their driving licenses for at least six years and have their own cars. Test persons with spectacles were not permitted, as the spectacles highly influence the reflected light rays from the two eyes. In addition, the eyebrows would be covered by the spectacle frame and would not be visible to the system. All subjects had sufficiently recognizable facial features that were not covered by hair or beard. They did not receive any special instructions for their driving style. They should drive as usual and always take into account the Road Traffic Regulations in Germany.

\section{Results}

As can be seen from the literature analysis, the visual patterns in left and right curves and on straight sections are very different. Since the gaze behavior when driving on straight sections is characterized by an accumulation of fixations on non-traffic-relevant road 
elements, [5] states that the driver's gaze on straight lines is almost evenly distributed over the entire width of the road and is even aligned towards the sky. This statement could be confirmed in this research. As can be seen in figure 3, the number of fixations on straight sections to the left and right of the main visual axis is almost the same. $46.7 \%$ of all fixations are registered to the left of the main gaze axis and 52.3\% are to the right. A large number of fixations are above the horizon. Only $1.0 \%$ of the fixations are exactly in the main gaze direction above and below the horizontal axis. No differences were found between the mean fixation durations on both sides. Looking at all fixations, their mean fixation location is about $1.0^{\circ}$ to the right of the main gaze direction. This finding confirms the finding of [1] that on straight sections the gaze is directed straight in the area of the vanishing point and the main fixation point is at approx. $1.6^{\circ}$ to the right of the main gaze direction.

Through the calculation of the average positions of the fixations, which were on the left and on the right side of the main visual direction, a significant difference could be found. While the average position of all left fixations is $2.5^{\circ}$ on the left side of the main visual axis, the average position of the right fixations is $4.0^{\circ}$ to the right of the main visual axis. Also, the standard deviation of the gaze angle from the main view direction in the horizontal direction is smaller on the left side than that on the right of the main view axis. This means that there is a greater gaze dispersion on the right side of the main direction compared to the left side. This visual pattern, which is common to all subjects, serves both to ensure correct vehicle position and lane tracking and to recognize the objects required for handling and orientation (for example traffic signs). The vertical arrangement of the fixations at $15^{\circ}-16^{\circ}$ on the right clearly shows the observation of the traffic signs.

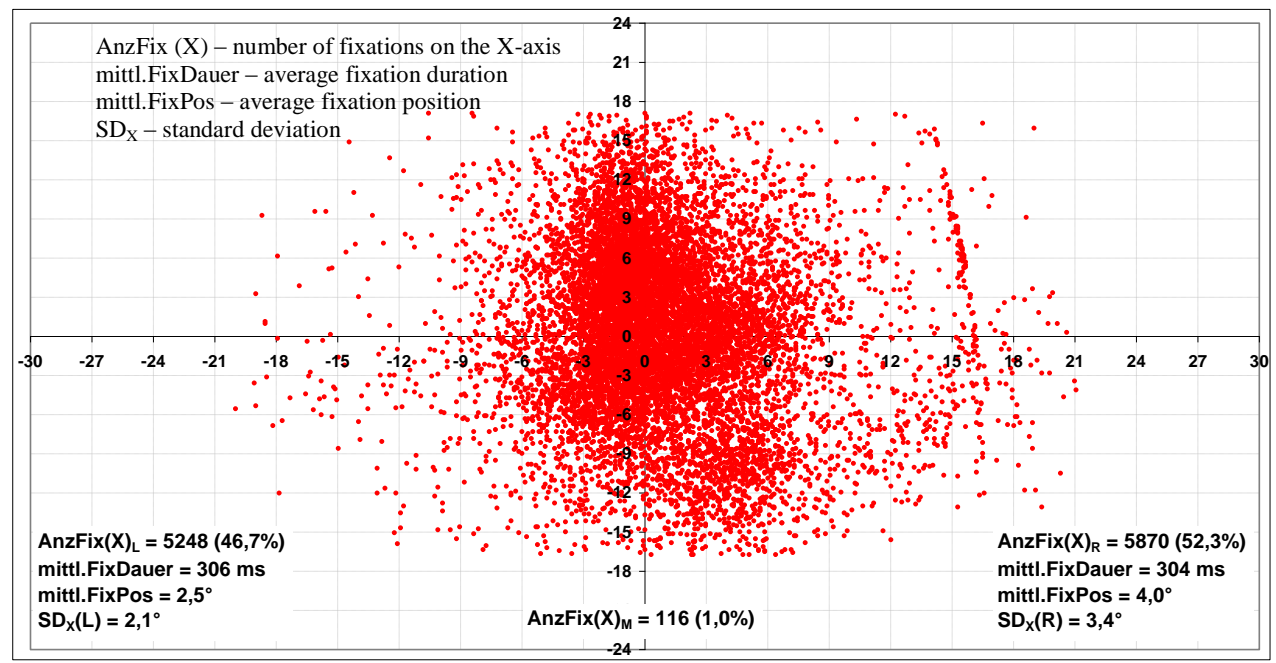

Fig. 3. Fixation distribution on straight sections.

In left curves (figure 4), the number of fixations on the left side of the main gaze axis is significantly larger $(67.5 \%)$ than on the right side $(32.1 \%)$. The average fixation duration is the same on both sides, but it is smaller than on straight sections. The average fixation positions are in almost equal distance on both sides of the main gaze axis with $3.9^{\circ}$ on the right side and $4.1^{\circ}$. The standard deviations in the lateral direction are also almost identical on the two sides of the main viewing axis. This leads to the conclusion that in left curves the gaze scatters equally on both sides of the main gaze axis, despite a stronger concentration of fixations on the left side. Thus, the findings of [1], [2] and [5] that in left curves the gaze is directed mainly straightforward and the fixation points are distributed 
over the entire width of the motorway, could be verified. Accordingly, the information in left curves is obtained from the entire road width. As on straight road sections, the vertical alignment of the fixation points at $15^{\circ}-16^{\circ}$ to the right of the main visual axis shows the targeted observation of traffic signs.

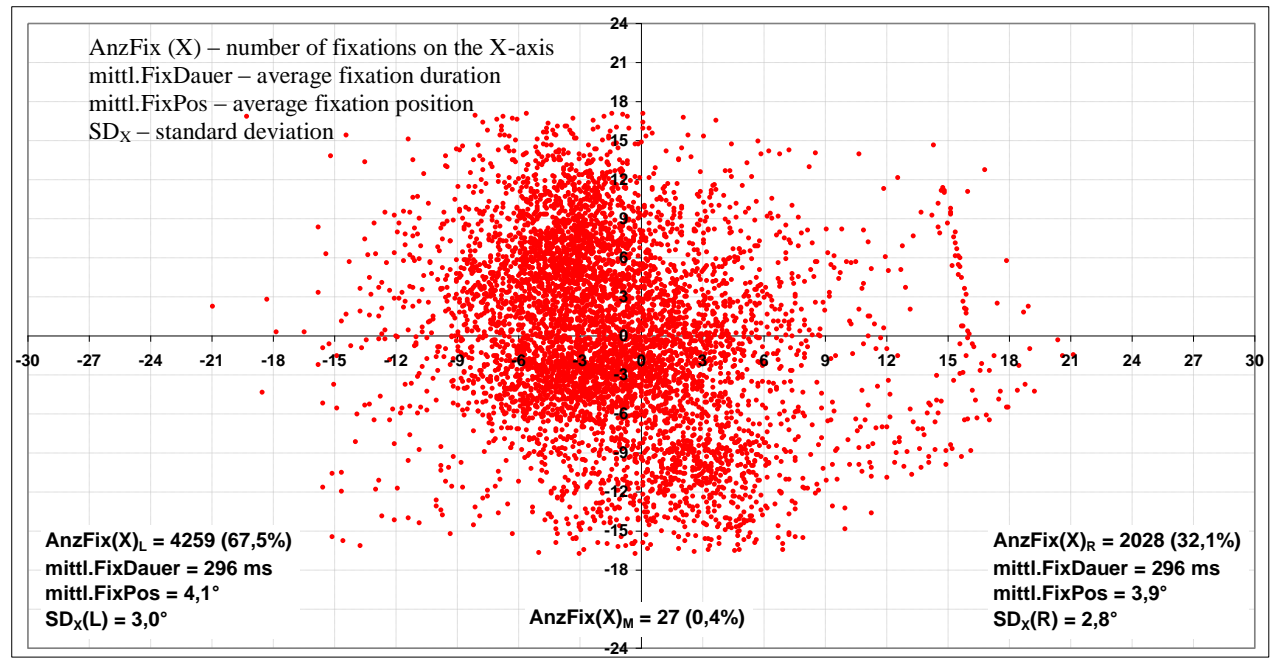

Fig. 4. Fixation distribution in left curves.

In right curves, on the other hand, drivers clearly fixate mainly on the right side of the main gaze direction (figure 5), where the vast majority of fixations take place (78.9\%). The gaze does not scatter over the entire roadway width as it does in left curves. While in right curves only $20.3 \%$ of all fixations can be registered on the left side of the gaze axis, the percentage of fixations on the right side in left curves is $32.1 \%$. The average fixation duration in right curves is longer $(312 \mathrm{~ms})$ than the one in left curves $(296 \mathrm{~ms})$. It was found that the fixations on the left side of the visual field in right curves account for about $9 \%$ of the total time. In contrast, in left curves drivers fixate about $18 \%$ of the time to the right side of the main gaze axis. Most of the relevant information is obtained by the driver from the areas of the right edge of the road and the vanishing point. Only a few glances arrive on the left side of the main viewing axis and they are mainly for orientation. From the video recordings in this study, the fixation points were counted and it was found that over $60 \%$ of all fixations reach the area of the right edge of the track at different distances. The average fixation position is found to be $4.5^{\circ}$ on the right side and only $2.4^{\circ}$ on the left side of the main gaze axis.

In right curves, drivers only look $2.4^{\circ}$ to the left, while they fixate $3.9^{\circ}$ to the right in left curves. The standard deviations on the two sides of the main gaze axis clearly show that the variation of the gaze in right curves on the left side is relatively small compared to the right side (figure 5). On the contrary, the gaze scatters almost equally on both sides of the gaze axis in left curves (figure 4). Thus, the right side is relevant for the lane tracking, the control of the vehicle and the information acquisition. 


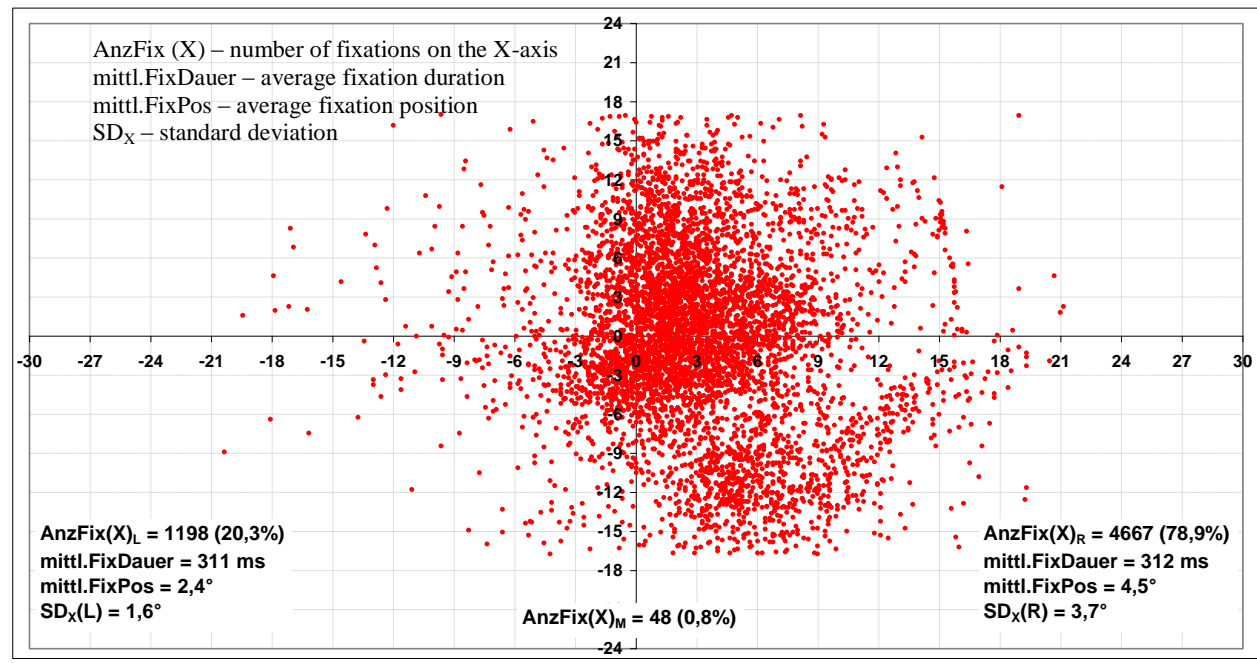

Fig. 5. Fixation distribution in right curves.

Figure 6 shows that drivers fixate much more near the vanishing point in curves than on straight sections. Driver's stress state is relatively low on straight roads and many traffic irrelevant objects are fixated, which is one of the reasons for the low concentration of fixations near the visual road vanishing point. The vanishing point remains in an unchanged position and serves as a constantly observed orientation point for the long-distance orientation, the driving direction and the lane tracking. As soon as drivers notice an approaching curve, they use their central vision for the information recording of trafficrelevant road elements such as lane markings and vanishing point. The higher number of fixations in the area around the vanishing point in both left and right curves shows that significantly higher PRC values are to be expected as a result of increasing stress in curves than on straight sections.

Because of the large distribution of fixations across the entire road width in left curves, the PRC value is much smaller than in right curves (figure 7). In right curves, the percentage of fixations in the area of the vanishing point is therefore the highest.

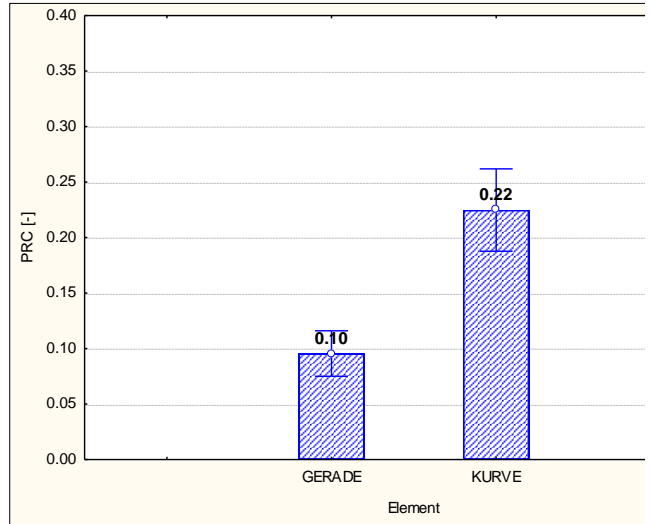

Fig. 6. Percentage of fixations to the main attention area (PRC) on straight sections and curves.

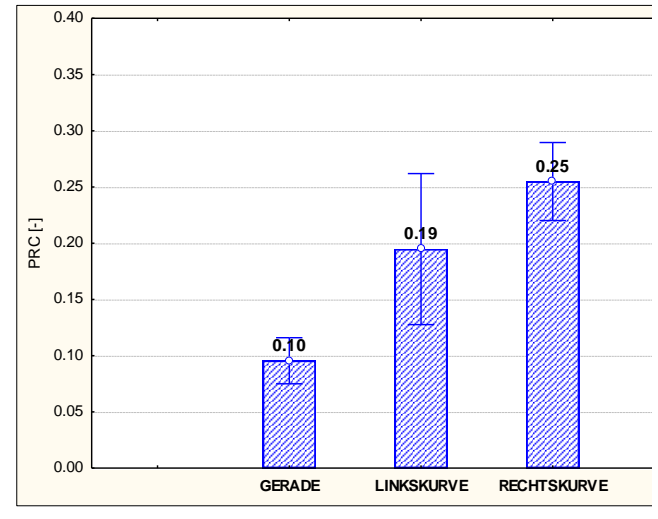

Fig. 7. Percentage of fixations to the main attention area (PRC) on straight sections, left and right curves. 


\section{Conclusions}

In this research on an existing motorway in Germany it has been found, that the driver's gaze on straight lines is almost evenly distributed over the entire width of the road. The necessary information for the orientation, the driving direction and the lane tracking in left curves is obtained from the entire road width. In right curves, the right edge of the road is preferably fixed and mainly the right side of the main gaze direction is relevant for the lane tracking, the control of the vehicle and the information acquisition. Compared to straight sections, a smaller gaze scatter could be established when driving in curves. However, information is searched more intensively in curves. The PRC parameter provides clear results and shows that drivers fixate more near the optical vanishing point in curves. This finding leads to the conclusion that there is an increased stress and concentration of the gaze in horizontal curves compared to straight sections.

\section{References}

1. Shinar D et al Sze S M 1977 Eye movements in curve negotiation (Human Factors, 19(1), 63 - 71, 1977)

2. Friedinger Ch 1982 Informationsaufnahme und Fahrverhalten (Straße und Verkehr, Heft 10)

3. Blaauw G J 1975 Drivers' scanning behavior on some curved and straight road sections (First International Congress on Vision and Road Safety, Paris, France)

4. Berger H 1996 Informationsaufnahme im Straßenverkehr - Grundlagen und Konsequenzen für die Praxis (Promotion an der Universität für Bodenkultur Wien, Institut für Verkehrswesen)

5. Cohen A S 1987 Blickverhalten und Informationsaufnahme von Kraftfahrern (Bericht zum Forschungsprojekt FE 8306/3 im Auftrag der BASt, ETH Zürich)

6. Underwood $\mathrm{G}$ et al 2002 Visual search while driving: skill and awareness during inspection of the scene (Transportation Research Part F: Traffic Psychology and Behavior, 5, 87-97)

7. Boer E R and Mulder M 2002 To brake or not to brake: Scaling the curve (A.G. Gale et al. (Hrsg.), Vision in Vehicles IX, Amsterdam: Elsevier)

8. Land M F and Horwood J 1995 Which parts of the road guide steering? (Nature 377, $339-340)$

9. Yorozu N et al 1999 Study on Driver Eye Fixation Points on Curved Road (Proceedings of 6th World Congress on Intelligent Transport Systems)

10. Popieul J-C et al 2000 Car driver eye scanning behavior: Study during a long duration highway travel in a simulator (Driving Simulation Conference, 6-7 September, Paris, France)

11. Boff K R and Lincoln J E 1988 Engineering data compendium - Human perception and performance (Armstrong Aerospace Medical Research Laboratory, Wright-Patterson Airforce Base, Ohio)

12. Unema P J A 1995 Eye movements and mental effort (Aachen)

13. Young L R and Sheena D 1975 Survey of eye movement recording methods (Behavior Research Methods \& Instrumentation, 7)

14. Karsch R and Breitenbach F W 1983 Looking at the amorphous fixation measure (Eye movements and psychological functions, Hillsdale, NJ)

15. Schweigert M 2003 Fahrerblickverhalten und Nebenaufgaben (Dissertation TU München) 